\title{
Eröffnungsansprache zum Ersten Internationalen Symposion über Probleme der quantitativen Biologie des Stoffwechsels, Helgoland, 1963
}

\author{
Otto KInNe
}

\author{
Biologische Anstalt Helgoland, Zentrale, Hamburg-Altona
}

Meine sehr verehrten Damen und Herren!

Im Namen des Organisationskomitees des Ersten internationalen Symposions über Probleme der quantitativen Biologie des Stoffwechsels spreche ich Ihnen meinen Dank aus dafür, daß Sie der Einladung zu dieser internationalen Veranstaltung Folge geleister haben. Es ist eine große Ehre und Freude für mich, Sie hier in der Meeresstation der Biologischen Anstalt Helgoland auf das herzlichste willkommen zu heißen. Insbesondere begrüße ich die Kollegen aus USA und Kanada, die den weiten Weg über den "großen Teich" nicht gescheut haben.

In zahlreichen Forschungszweigen der modernen Biologie und Medizin haben die letzten Jahre viele tiefgreifende, neue Erkenntnisse über quantitative Aspekte des Stoffwechselgeschehens erbracht. Es ist nunmehr an der Zeit, diese neuen Informationen auf breiter Basis in einer interdisziplinären Zusammenkunft eingehend zu erörtern. Wir sind glücklich darüber, daß so viele namhafte Biologen und Mediziner sich zu diesem Erfahrungs- und Gedankenaustausch zusammengefunden haben - eine „Elitegruppe", wie Herr vON BERTALANFEx mir vorhin versicherte.

Bitte lassen Sie mich auch an dieser Stelle zwei Kollegen herzlichst danken für ihre großen Verdienste an dem Zustandekommen dieses Symposions: Herrn Dr. A. Locker, Wien, und Herrn Professor F. Krüger, Hamburg. Sie sind die geistigen Väter dieses Symposions. Mein Dank gilt ferner jenen Behörden und Institutionen, welche diese Zusammenkunft durch finanzielle Zuwendungen ermöglicht haben. Ich nenne hier vor allem das Bundesministerium für Ernährung, Landwirtschaft und Forsten, Bonn, die Hoffmann-La Roche G.m.b.H., Wien-Basel, und die Ósterreichische Studiengesellschaft für Atomenergie G.m.b.H., Wien. Last not least möchte ich der Gemeinde Helgoland und ihrem Bürgermeister, Herrn Rickmers, wärmstens Dank sagen für die zahlreichen Beweise ihrer freundschaftlichen Verbundenheit mit der Biologischen Anstalt Helgoland.

Es ist kein Zufall, daß wir uns hier auf Helgoland zusammenfinden. Das neue Forschungsprogramm der Biologischen Anstalt Helgoland mißt den quantitativen Veränderungen biologischer Systeme, vor allem im Hinblick auf die modifizierende 
Wirkung der Umweltfaktoren, große Bedeutung bei. Innerhalb des wissenschaftlichen Stabes unserer Anstalt hat eine Forschungsgruppe gerade damit begonnen, sich einer eingehenden, langfristigen Analyse der quantitativen Parameter der Aufbau-, Umbauund Abbauvorgänge im Meer zu widmen. Zudem ist Helgoland ein idealer Tagungsort. Da ist einmal die gemeinsame Seefahrt auf dem schmucken Bäderschiff, welche die persönliche Begegnung erleichtert, ja stimuliert. Auf der Insel angekommen, begrüßen den Besucher moderne, formschöne Häuser und Anlagen und vermitteln vor dem Hintergrund der roten Felsen eine Atmosphäre der Harmonie und Aufgeschlossenheit. Mit Recht sagen die Helgoländer: "Auf Helgoland ist alles anders."

Auch in biologischer Hinsicht kommt der Insel eine Sonderstellung zu. Innerhalb der Grenzen der Bundesrepublik erreichen Reichtum und Formenfülle der marinen Fauna und Flora hier ihren Höhepunkt. Diese Besonderheit hat seit mehr als 100 Jahren führende Naturwissenschaftler angezogen. 1835 entdeckte EHRENBERG auf Helgoland die Ursache des Meeresleuchtens in dem einzelligen Flagellat Noctiluca miliaris. Von 1845 bis 1854 wirkte hier Johannes Müller. Er erschloß den Naturwissenschaftlern die Wunderwelt des Planktons. MüLLER veröffentlichte zahlreiche grundlegende Arbeiten über die Larvenformen verschiedener Tiergruppen und über die Entwicklung der Echinodermen. Wenig später arbeitete auf Helgoland eine Reihe prominenter und heute weltberühmter Forscher. Ich nenne hier nur ERnst HAEckel, den „Vater" der Okologie, und ANton Dohrn, den Begründer der Zoologischen Station in Neapel. Unlösbar sind viele andere klangreiche Namen mit der Gründung, Geschichte und Arbeit der Biologischen Anstalt Helgoland verknüpft: Braun, Leuckart, Pagenstecher, Pringshem, Sachs, H. A. Meyer, Oppenheim, Heincke, Mielck, Hagmeier, Hertling, Kuckuck, Nienburg, Ehrenbaum, Schreiber, dessen „Schreiber"und "Erdschreiberlösungen" heute weltbekannt sind, und andere.

Der gemeinsame Wunsch zahlreicher deutscher Naturwissenschaftler nach einer ständigen Forschungsstätte für meeresbiologische Untersuchungen auf Helgoland fand seinen Ausdruck in einem Antrag zur Errichtung einer Zoologischen Station auf Helgoland, welcher 1876 anläßlich einer Tagung der Deutschen Naturforscher und Arzte in Hamburg gestellt wurde. Im April des Jahres 1892 war es dann endlich soweit: das preußische Kultusministerium gründete die "Königliche Biologische Anstalt auf Helgoland". Es ist mir hier nicht möglich, die Geschichte und Entwicklung unserer Anstalt im einzelnen darzulegen. Ich möchte Ihnen aber in aller Kürze über die gegenwärtige Situation berichten.

Die beiden heutigen Hauptaufgabenbereiche der Biologischen Anstalt Helgoland lassen sich wie folgt umreißen: 1. Grundlagenforschung auf allen Gebieten der Meeresbiologie, 2. Förderung der Kenntnis vom Leben im Meer, und zwar durch: a) Bereitstellung von Arbeitsplätzen für Gastforscher, b) Veranstaltung meeresbiologischer Kurse, c) Versorgung festländischer Lehr- und Forschungsstätten mit marinem Untersuchungsmaterial und d) Unterhaltung eines Schau-, Lehr- und Versuchsaquariums. Zur Durchführung dieser Aufgaben verfügt die Anstalt gegenwärtig über einen Stab von insgesamt 17 Wissenschaftlern und 53 technischen und administrativen Hilfskräften sowie über einen seegehenden Forschungskutter, die "Uthörn“, und mehrere kleinere Boote für den Stationsbetrieb auf Helgoland und Sylt. Der wissenschaftliche Stab gliedert sich in 7 Abteilungen, nämlich: Zoologie, Botanik, Physiologie, Mikrobiologie, 
Planktonforschung, Ichthyologie und Strahlenbiologie. Die Einrichtungen der Anstalt befinden sich an drei verschiedenen Orten: die Zentrale in Hamburg-Altona, die Meeresstation auf Helgoland und die Litoralstation in List auf Sylt. Die wichtigsten Einrichtungen und die zahlreichen Spezialräume der Meeresstation werden wir Ihnen in einem anschließenden Rundgang vorführen. Die Anstalt verfügt auf Helgoland ferner über ein Züchtungslaboratorium am Südhafen, ein Gästehaus mit 25 Zimmern auf dem Oberland sowie 9 Wohnhäuser für Anstaltsangehörige.

Auf der Basis des bisher erarbeiteten Tatsachenmaterials wendet sich die Biologische Anstalt Helgoland gegenwärtig einem Zentralproblem der modernen Meeresbiologie zu, nämlich der eingehenden Erforschung der Dynamik ökologischer Beziehungsketten im Meer und deren Abhängigkeit von Umweltfaktoren. Ein viele Quadratkilometer umfassendes Gebiet innerhalb der Deutschen Bucht dient dabei als Modell, an dem Einzelheiten der Wirkung physikalisch-chemischer Veränderungen auf biologische Systeme in engen Zeitintervallen über Jahre verfolgt werden. Die sich im Verlaufe dieser Untersuchungen als für das OKosystem besonders wichtig erweisenden Organismen sollen dann später im Laboratorium einer experimentell-ökologischen und physiologischen Analyse unterworfen werden. Gleichzeitig werden Wissenschaftler der Anstalt an großen nationalen und internationalen Gemeinschaftsunternehmungen teilnehmen, wie zum Beispiel an Expeditionen in den tropischen Atlantik, in den Nordatlantik und in den Indischen Ozean. Von einer konsequenten Durchführung einer solchen umfassenden Analyse versprechen wir uns neue wichtige Einblicke in die quantitative und qualitative Dynamik der Aufbau-, Umbau- und Abbauvorgänge im Meer und eine Vertiefung unserer Einsichten in die Komplexität mariner Okosysteme. Neben diesem neuen "Schwerpunktprogramm" werden die verschiedenen Forschungsrichtungen innerhalb der einzelnen Abteilungen beibehalten werden.

Ich hoffe, daß diese kurze Skizze Ihnen einen Einblick zu geben vermochte in die Geschichte und Gegenwart unserer Anstalt.

Zum Abschluß meiner Ausführungen möchte ich dem Wunsche Ausdruck verleihen, daß dieses Symposion allen Teilnehmern eine Bereicherung ihrer Kenntnisse und Auffassungen bescheren und einen harmonischen Verlauf nehmen möge. In diesem Sinne erkläre ich das „Erste internationale Symposion über Probleme der quantitativen Biologie des Stoffwechsels" für eröffnet. 